\title{
Design and Control Self Balancing Robot
}

\author{
S Santoso ${ }^{1}, S$ Yuliananda ${ }^{2}$ \\ \{ santoso@untag-sby.ac.id ${ }^{1}$, subektiyuliananda@untag-sby.ac.id ${ }^{2}$ \}
}

${ }^{1,2}$ Department of Electrical Engineering, University of August 17, 1945, Surabaya, Indonesia

\begin{abstract}
Balancing robot is a mobile robot that has wheels on the right and left side, has the ability to maintain equilibrium. This robot is a development of an inverted pendulum models placed on a wheeled train. Gy521 mpu6050 3-axis gyroscope and accelerometer sensors are used to detect the slope as well as to detect angular velocity and to maintain the position of the robot in a position perpendicular to the earth surface. PID control method is used to control the DC motors. The average value of the measured angle on the sensor is $\mathrm{Y}=90, \mathrm{X}=$ $0.1, Z=44$. The determination of the value (tuning) of Proportional and Integral control parameters is done by trial and error. The GYROBOT mobile app created to control the direction of robot moves through the Bluetooth channel. This research is still not getting stability in the robot movement,
\end{abstract}

Keywords: Android, balancing robot, gyroscope, PID, robot.

\section{Introduction}

The development of robotics technology has made the higher the quality of human life. Currently, the development of robotics technology has been able to improve the quality and quantity of various industries. Robotics technology has also been reaching outside of entertainment and education for human beings[1], [2]. One way to increase the level of intelligence of a robot is to add a sensor, a control method even provide artificial intelligence in robots, robots that have intelligence One is a self-balancing robot. balance robot (robot balancer) is a robot that has two wheels on the right and left are not balanced when without a controller. Balance this robot is the development of a model of inverted pendulum (inverted pendulum) are placed on top of the train wheels.

Balance is the ability to maintain an upright position of equilibrium against an object. Equilibrium process usually also called stability control. Two wheels are in place it on the base surface and allow the robot chassis to maintain an upright position and moving toward the slope, forward, backward, rotating in an effort to keep the center of mass above the axle [3].

MPU-6050 Module is a module-core MPU-6050 sensor is capable of reading the tilt angle based on data from an accelerometer sensor and sensor gyroscope, this sensor is also equipped with temperature sensors that can be used to measure the temperature of surroundings, Sensor MPU-6050 contains a MEMS Accelerometer and a mutually integrated MEMS Gyro. This sensor is very accurate with internal hardware facilities 16 bit ADC for each channel. This sensor will capture the value of channel angle $\mathrm{X}, \mathrm{Y}$ and $\mathrm{Z}$ at the same time [4].

Android is an operating system with an open-source operating system. Linux-based Android is designed for touch screens mobile devices such as smartphones and tablet computers. Android was originally developed by Android, Inc. [5] 
Bluetooth is a wireless communication protocol that works on a radio frequency of 2.4 GHz[6][7]. HC-05 Bluetooth module is a Bluetooth module that can be a slave or master this is evidenced by can provide notification to the other pair, or another device that pairing a Bluetooth module to the $\mathrm{CH}-05$. HC-05 Bluetooth module comprised of 6 pin connector, each pin connector has a different function - following differences described the function of each pin of the Bluetooth HC-05 [4]

AVR microcontroller (Alf and Vegard's Risc processor) ATmega328 that uses RISC technology (Reduce Instruction Set Computing) which programs run faster because it only takes one clock cycle to execute an instruction program. microcontroller has been equipped with an internal ADC, internal EEPROM, Timer / Counter, PWM, analog comparator, and others.

PID controller (Proportional-Integral-Derivative controller) is a control feedback mechanism typically used in industrial control systems. A PID controller continuously calculates the value of error as the difference between the desired setpoint and measured process variables. The controller tried to minimize the error value each time by adjusting the control variables, such as the tap position control, damper, or power on the heating element, to a new value that is determined by the sum of the following is a block diagram PID

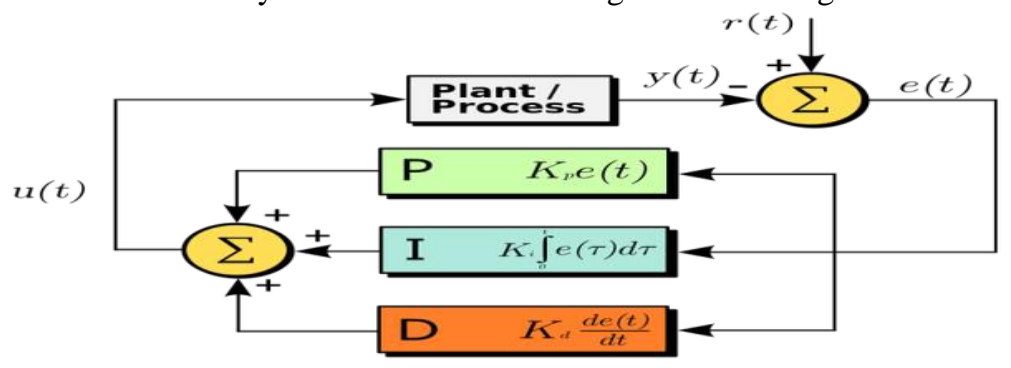

Figure 1. Block diagram of PID

The PID control equation as under this

$V o=K p \cdot e(t)+K i \int_{0}^{t} e(t) d t+K d \frac{d e(t)}{d t}$

Information :

$\mathrm{mv}(\mathrm{t})=$ output of the PID controller or Manipulated Variable

$\mathrm{Kp}=$ Constant Proportional

$\mathrm{Ti}=$ Constant Integral

$\mathrm{Td}=$ Constant Detivatif

e $(t)=$ Error (the difference between the set point with the actual level) [6] 


\section{Methodology}

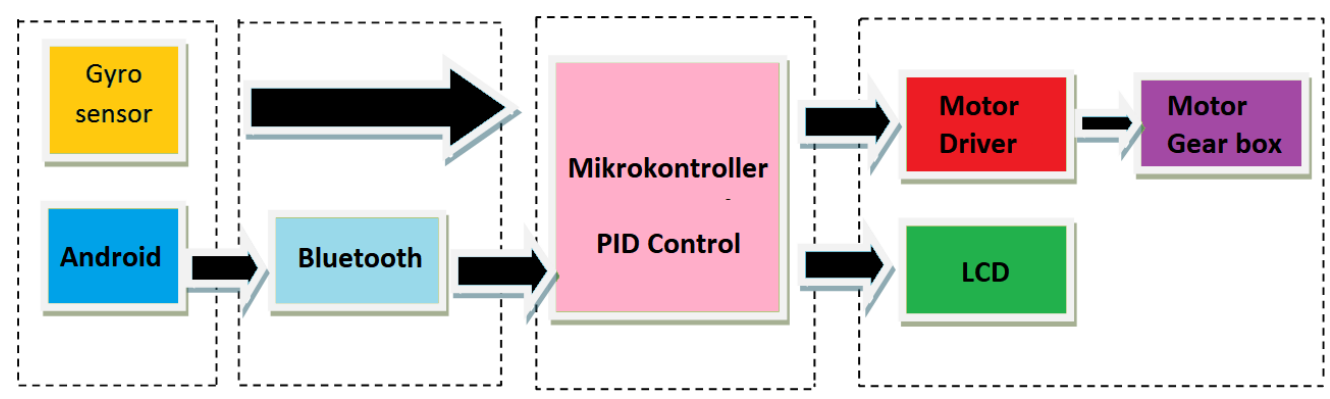

Figure 2. Diagram block balance robot

Gyro sensor: Enter the values of $\mathrm{x}, \mathrm{y}$, and $\mathrm{z}$ to a microcontroller. Android: Input data to the microcontroller triggers. Bluetooth: android media liaison to the microcontroller. Microcontroller Center balance control robot. the controller to determine the precision of a PID system.

Code program for Adjusting motor dc speed

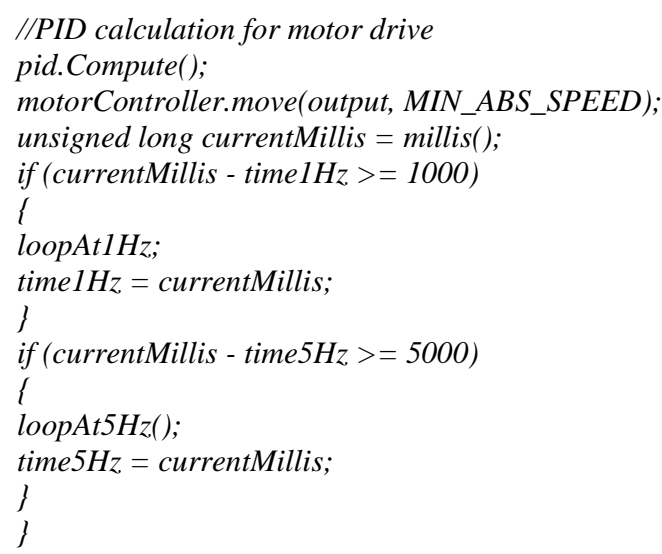

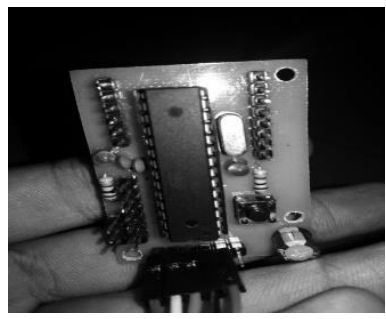

(a)

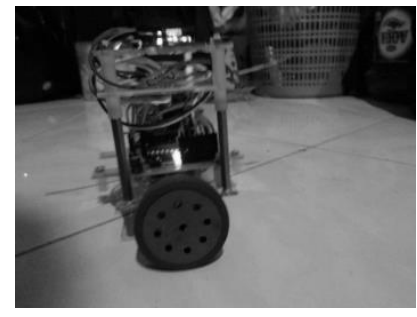

(b)

Figure 3. a) Minimum system ATmega 328, b) the robot balance position 


\section{Results and Discussion}

Gyro sensor testing aims to look at the value of the output which is derived from the sensor.

Table 1. Data Trailer gyrosensor mpu-6050

\begin{tabular}{ccc}
\hline $\mathrm{X}$ & $\mathrm{Y}$ & $\mathrm{Z}$ \\
\hline $3: 29$ & 71.91 & -11.46 \\
\hline $3: 26$ & 71.96 & -11.34 \\
\hline $3: 19$ & 72 & -11.22 \\
\hline $3: 17$ & 72.04 & -11.1 \\
\hline $3: 16$ & 72.07 & -10.99 \\
\hline $3: 13$ & 72.12 & -10.86 \\
\hline $3: 29$ & 71.91 & -11.46 \\
\hline $3: 26$ & 71.96 & -11.34 \\
\hline
\end{tabular}

Note: capture a snapshot of data serial arduino

\subsection{Testing Motor Driver}

Driver Motor function as a regulator of the direction of rotary motors de and de motor speed tested motor driver in the following ways:

Table 2. Setting the direction of rotary motors dc

\begin{tabular}{|c|c|c|c|c|c|c|}
\hline \multirow[t]{2}{*}{ Command } & \multicolumn{4}{|c|}{ Input Pin } & \multicolumn{2}{|c|}{ Direction dc Motor } \\
\hline & 1 & 2 & 3 & 4 & Left & Right \\
\hline Forward & 0 & 1 & 0 & 1 & 4 & 4 \\
\hline Reverse & 1 & 0 & 1 & 0 & & \\
\hline Left & 1 & 0 & 0 & 1 & $X$ & \\
\hline Right & 0 & 1 & 1 & 0 & & $\lambda$ \\
\hline
\end{tabular}

\subsection{Balance Testing Robot}

System testing is done after the program and downloads a list of programs into the microcontroller, to know that the entire system is designed to work well. To use the robot balance, there are several stages

Table 3. mpu-6050 data, when the robot balance stood to maintain an upright position

\begin{tabular}{ccc}
\hline $\mathbf{X}$ & $\mathbf{Y}$ & $\mathbf{Z}$ \\
\hline $\mathbf{9 0}$ & $1: 51$ & -0.23 \\
\hline $\mathbf{9 0 . 0 8}$ & 1.64 & -0.26 \\
\hline $\mathbf{9 0 . 1 5}$ & 1.7 & -0.31 \\
\hline $\mathbf{9 0 . 2 2}$ & 1.73 & -0.37 \\
\hline $\mathbf{9 0 . 2 8}$ & 1.76 & -0.42 \\
\hline
\end{tabular}




\begin{tabular}{lll}
\hline $\mathbf{9 0 . 3 3}$ & 1.82 & -0.47 \\
\hline $\mathbf{9 0 . 2 8}$ & 1.76 & -0.42 \\
\hline $\mathbf{9 0 . 0 8}$ & 1.64 & -0.26
\end{tabular}

\subsection{Android Application Testing}

Android app has been created using Appinventor2 in test by downloading applications that have been made and try to connect to a Bluetooth device HC-05, after connecting the input voice commands to turn to balance the robot when the robot responds to commands from the application, there will be feedback in the form of a voice of the android application.

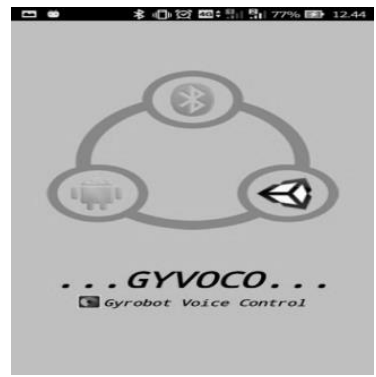

Figure 4. Application on android

\section{Conclusion}

Based on the measurement results and concluded Balance control robot with a gyro sensor and android is working properly and able to stand upright, communication robot and smartphone utilizing i2c data path. Gyro sensors capable of processing input to balance the communication between the robot and the android and the microcontroller using Bluetooth successfully use the data lines RX and TX to trigger the start of robots, but to control the direction of motion of the robot is still a difficulty for synchronous commands from android and gyro sensor to the dc motor.

\section{References}

[1] T. Shibata, "An overview of human interactive robots for psychological enrichment," Proc. IEEE, vol. 92, no. 11, pp. 1749-1758, Nov. 2004.

[2] T. Shibata and K. Wada, "Robot Therapy: A New Approach for Mental Healthcare of the Elderly \&\#150; A Mini-Review," Gerontology, vol. 57, no. 4, pp. 378-386, 2011.

[3] A. Lieu, "Two-Wheeled Balancing Robot Using Integral KendaliProporsional Method," Semarang.

[4] B. Ave, "mpu data sheet 6050," www.invensense.com, 2013. .

[5] Iteadstudio.com, "HC bluetooth data sheet 05.," http://www.electronicaestudio.com/docs/istd016A.pdf, 2010. .

[6] E. Ferro and F. Potorti, "Bluetooth and wi-fi wireless protocols: a survey and a 
comparison," IEEE Wirel. Commun., vol. 12, no. 1, pp. 12-26, Feb. 2005.

[7] V. K. Garg, "An Overview of Wireless Systems," in Wireless Communications \& Networking, Elsevier, 2007, pp. 1-21. 